\title{
An Unprecedented Ring Transformation of a 4-(Aminomethyl)oxazoline Derivative to a 4-(Hydroxymethyl)imidazoline
}

\author{
Marvin Schulz, Jens Christoffers* \\ Institut für Chemie, Carl von Ossietzky Universität Oldenburg, 26111 Oldenburg, Germany \\ Fax +49(441)7983873; E-mail: jens.christoffers@uni-oldenburg.de \\ Received: 27.08.2013; Accepted after revision: 30.09.2013
}

\begin{abstract}
An optically active 4-(azidomethyl)-2-(2-pyridyl)oxazoline was prepared starting from L-serine and picolinic acid. Reduction of the azide moiety gave the corresponding 4-(aminomethyl)2-(2-pyridyl)oxazoline, which is not a stable compound but readily undergoes ring-transformation rearrangement to furnish a 4-(hydroxymethyl)-2-(2-pyridyl)imidazoline. After Boc protection of the amidine function, the material could be further converted into the 4(aminomethyl)-2-(2-pyridyl)imidazoline via the respective azidomethyl compound.
\end{abstract}

Key words: heterocycles, oxazolines, imidazolines, pyridines, chiral ligands

Since the first reports by Brunner and co-workers, ${ }^{1}$ optically active 2-pyridyloxazolines have become a privileged class of chiral ligands in asymmetric catalysis. ${ }^{2,3}$ In continuation of our earlier work on $C_{1}$-symmetric, tridentate pyridyloxazolines, ${ }^{4}$ we were planning to prepare compound 1 with an aminomethyl group, the latter being perfectly suited for further $\mathrm{N}$-functionalization, for example by amidation reactions. In the event, we were unable to isolate compound $\mathbf{1}$, nor its derivatives, as it always underwent ring transformation to furnish the hydroxymethyl-functionalized pyridylimidazoline 2 (Scheme 1). Such a ring transformation has been reported once before for the formation of a cyclic urea from a urethane. ${ }^{5}$ It has been at least suspected for [ $(N$-arylamino $)$ methyl $]$ oxazolines, ${ }^{6}$ but not observed by others, although some researchers claim to have isolated an (aminomethyl)oxazoline as an intermediate product. ${ }^{7}$ In one other case, an in situ formed (aminomethyl)oxazoline was protected with $\mathrm{CbzCl}$ in the reaction mixture. ${ }^{8}$ Since optically active imidazolines are an increasingly important, novel class of chiral ligands, ${ }^{9}$ investigations on further conversion of the product 2 seemed promising to us. Therefore, we now report on the preparation of compound $\mathbf{1}$ and its transformation to imidazoline $\mathbf{2}$, as well as some further chemistry starting with the latter compound.
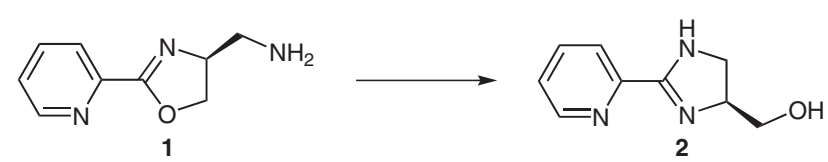

Scheme 1 Facile ring transformation of 4-(aminomethyl)oxazoline 1 to 4-(hydroxymethyl)imidazoline 2

SYNTHESIS 2014, 46, 0081-0086

Advanced online publication: 04.11 .2013

DOI: 10.1055/s-0033-1340044; Art ID: SS-2013-T0591-OP

(C) Georg Thieme Verlag Stuttgart · New York
The starting point of our investigation was the hydroxymethyl compound 7 , which was obtained by the five-step sequence outlined in Scheme 2. Picolinic acid (3) was first coupled with methyl L-serinate ${ }^{10}$ to give amide $4(71 \%) .{ }^{11}$ The primary alcohol was then TBS protected $(92 \%$ yield of compound $\mathbf{5})^{12}$ and the ester moiety reduced to again give a primary alcohol 8 (79\%), which was activated with tosyl chloride and cyclized according to a literature protocol. ${ }^{13}$ Intermediate product $6(77 \%)$ was finally deprotected with tetrabutylammonium fluoride $(93 \%$ yield of product 7).

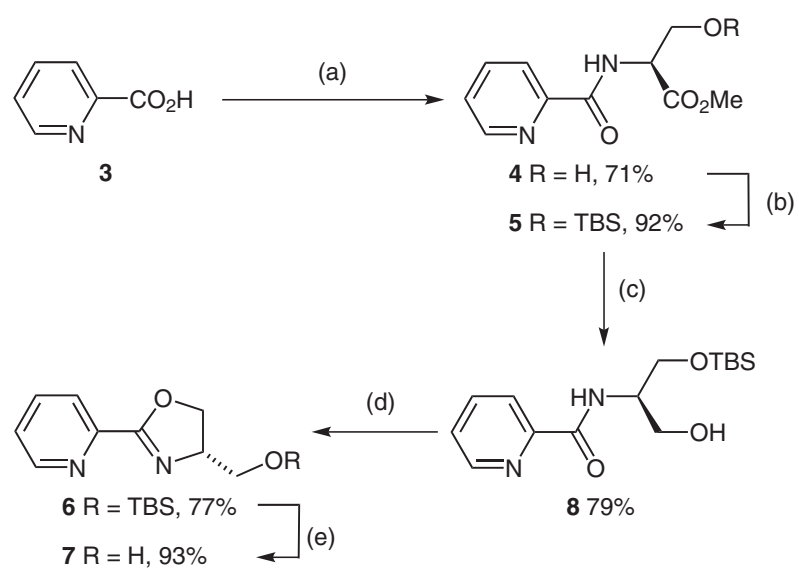

Scheme 2 Preparation of hydroxymethyl compound 7. Reagents and conditions: (a) NMM (2.3 equiv), $\mathrm{ClCO}_{2} \mathrm{Et}$ (1.1 equiv), (S)$\mathrm{MeO}_{2} \mathrm{CCH}\left(\mathrm{CH}_{2} \mathrm{OH}\right) \mathrm{NH}_{2} \cdot \mathrm{HCl}$ (1.1 equiv), THF, $0{ }^{\circ} \mathrm{C}$ to r.t., $2 \mathrm{~h}$; (b) TBSCl (1.3 equiv), imidazole (3 equiv), $\mathrm{CH}_{2} \mathrm{Cl}_{2}$, r.t., $1 \mathrm{~h}$; (c) $\mathrm{LiBH}_{4}$ (1.3 equiv), THF, $0{ }^{\circ} \mathrm{C}, 3 \mathrm{~h}$; (d) TsCl (1.2 equiv), DMAP (0.1 equiv), DCE, reflux, $16 \mathrm{~h}$; (e) TBAF $3 \mathrm{H}_{2} \mathrm{O}$ (1.2 equiv), THF, r.t., $1 \mathrm{~h}$; TBS = $t-\mathrm{BuMe}_{2} \mathrm{Si}$, Ts $=4-\mathrm{MeC}_{6} \mathrm{H}_{4} \mathrm{SO}_{2}$.

Organoazides are excellent precursors for primary amines; therefore, we activated the primary alcohol function of compound 7 by sulfonyl ester formation $(95 \%$ of product 9) and converted it with sodium azide in ethanol following common protocols (Scheme 3) ${ }^{14}$ Product 10, after chromatography, was obtained in almost quantitative yield. Azide 10 was subjected to catalytic hydrogenation to again give a single compound (95\% yield) with correct mass (ESI) and ${ }^{1} \mathrm{H}$ and ${ }^{13} \mathrm{C}$ NMR spectra that seemed to be in agreement with structure 1; however, all attempts at further derivatization of what we assumed to be an amino function, in particular amide formation, failed. We realized that the ring-transformation reaction of intermediate product 1 with formation of imidazoline 2 would be an explanation for our failure to produce amides of purported 
amine 1. Imidazoline 2 would give similar ${ }^{1} \mathrm{H}$ and ${ }^{13} \mathrm{C}$ NMR spectra, leading us astray. This rearrangement appears obvious, since the amino group as a good nucleophile is at the right distance to form a bicyclo[2.2.1] intermediate structure when adding to the electrophilic imidoester group.

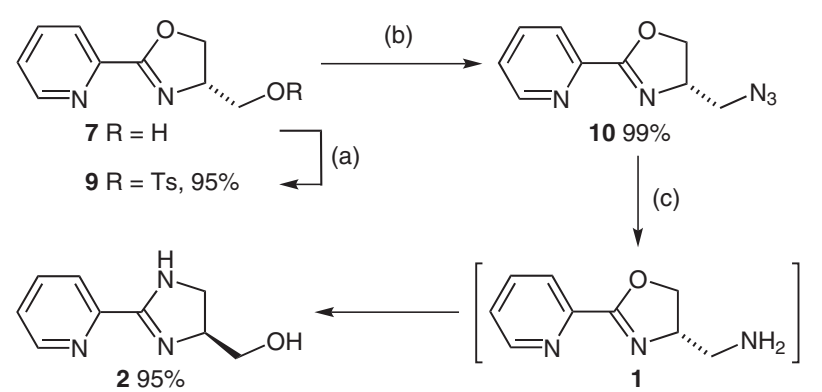

Scheme 3 Preparation and ring transformation of 4-(aminomethyl)oxazoline 1. Reagents and conditions: (a) $\mathrm{TsCl}$ (1.5 equiv), $\mathrm{KOH}$, $\mathrm{H}_{2} \mathrm{O}, \mathrm{CH}_{2} \mathrm{Cl}_{2}$, reflux, $3 \mathrm{~h}$; (b) $\mathrm{NaN}_{3}$ (5 equiv), EtOH, reflux, $16 \mathrm{~h}$; (c) $\mathrm{H}_{2}$ (1 atm), $\mathrm{Pd} / \mathrm{C}$ (cat.), $\mathrm{MeOH}, 23^{\circ} \mathrm{C}, 16 \mathrm{~h}$.

Definite proof of the structure of compound 2 came from its subsequent chemistry, which was initially difficult as the very basic amidine function caused several problems with chemoselectivity. These problems could, however, be solved by reducing the electron density of 2 by installing a carbamate function (product 11, 67\% yield of crude material; Scheme 4). Interestingly, we obtained a single compound, although one would expect a regioselectivity problem due to the presence of two nucleophilic nitrogen atoms at the amidine function. The depicted regiochemistry was proven by 2D-NMR spectroscopy, after the assignment of all resonances. Firstly, C-4 and 4-H were identified by DEPT 135 and HMQC experiments $\left[\delta\left({ }^{13} \mathrm{C}\right)=\right.$ $\left.66.8, \delta\left({ }^{1} \mathrm{H}\right)=4.28 \mathrm{ppm}\right]$. Furthermore, the pyridine signals were identified by $\mathrm{H}, \mathrm{H}-\mathrm{COSY}$ and $\mathrm{HMQC}$ experiments, as follows $\left[\delta\left({ }^{1} \mathrm{H}\right), \delta\left({ }^{13} \mathrm{C}\right)\right.$, in ppm]: $7.5\left(3^{\prime}-\mathrm{H}\right), 123.4(\mathrm{C}-$ 3'); 7.7 (4'-H), 136.3 (C-4'); 7.29 (5'-H), $124.3\left(\mathrm{C}-5^{\prime}\right)$; $8.58\left(6^{\prime}-\mathrm{H}\right), 148.8\left(\mathrm{C}-6^{\prime}\right)$. The quaternary pyridine $\mathrm{C}-2^{\prime}$ was then identified at $151.4 \mathrm{ppm}$ (HMBC cross peaks with $6^{\prime}-\mathrm{H}$ and $\left.4^{\prime}-\mathrm{H}\right)$. Of the two remaining $\mathrm{sp}^{2}$-carbon resonances, the amidine $\mathrm{C}-2$ was assigned at $\delta=159.4 \mathrm{ppm}$ by the HMBC cross peak with 3 '-H. Therefore, the carbamate $\mathrm{C}=\mathrm{O}$ must be the remaining sp ${ }^{2}$ signal at $\delta=150.4 \mathrm{ppm}$. The two methylene protons $5-\mathrm{H}$ were then identified at $\delta=3.84$ and $3.99 \mathrm{ppm}$ by the HMBC cross peak with the amidine $\mathrm{C}-2$; the respective $\mathrm{C}-5$ was identified at $\delta=49.3$ ppm (HMQC experiment). The remaining $4-\mathrm{CH}_{2} \mathrm{OH}$ was identified at $\delta\left({ }^{1} \mathrm{H}\right)=3.64$ and $3.74 \mathrm{ppm}$, and $\delta\left({ }^{13} \mathrm{C}\right)=64.3$ ppm (HMQC experiment). With this assignment of all proton and carbon resonances in hand, the structure of compound 11 was finally proven by ${ }^{15} \mathrm{~N},{ }^{1} \mathrm{H}-\mathrm{HMBC}$ spectroscopy; three ${ }^{15} \mathrm{~N}$ signals were observed, and were assigned according to the literature data for a similar 1acetyl-2-aryl-4,5-dihydroimidazole: ${ }^{15} \delta\left({ }^{15} \mathrm{~N}\right)=-74.1(\mathrm{~N}-$ $\left.1^{\prime}\right),-122.8(\mathrm{~N}-3),-244.5(\mathrm{~N}-1) \mathrm{ppm}$, with $\mathrm{MeNO}_{2}(\delta=0)$ as external standard. Naturally, within the pyridine ring, $\mathrm{N}-1^{\prime}$ showed cross peaks to $3^{\prime}-\mathrm{H}, 5^{\prime}-\mathrm{H}$ and $6^{\prime}-\mathrm{H}$. The car- bamate $\mathrm{N}-1$ showed three strong cross peaks, to $4-\mathrm{H}$ and both $5-\mathrm{H}$ protons, whereas the imine $\mathrm{N}-3$ showed two strong cross peaks, to both $4-\mathrm{CH}_{2} \mathrm{OH}$ protons, and one weak cross peak, to one $5-\mathrm{H}$ proton.

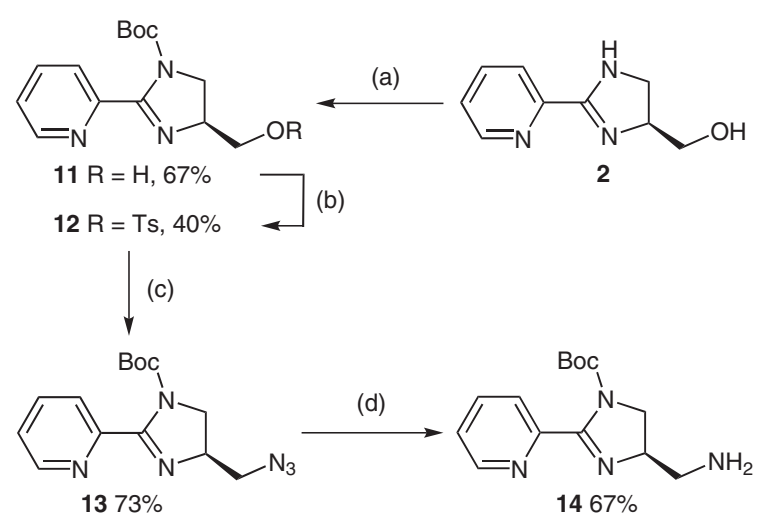

Scheme 4 Preparation and reduction of azide 13. Reagents and conditions: (a) $\mathrm{Boc}_{2} \mathrm{O}$ (2.5 equiv), $\mathrm{K}_{2} \mathrm{CO}_{3}$ (2 equiv), $\mathrm{H}_{2} \mathrm{O}$, THF, reflux, 24 h; (b) $\mathrm{TsCl}$ (6.3 equiv), $\mathrm{KOH}, \mathrm{H}_{2} \mathrm{O}, \mathrm{CH}_{2} \mathrm{Cl}_{2}$, reflux, $3 \mathrm{~h}$; (c) $\mathrm{NaN}_{3}(5$ equiv), EtOH, reflux, $16 \mathrm{~h}$; (d) $\mathrm{H}_{2}$ (1 atm), $\mathrm{Pd} / \mathrm{C}$ (cat.), $\mathrm{MeOH}, 23{ }^{\circ} \mathrm{C}$, $16 \mathrm{~h}$.

The alcohol function of $\mathbf{1 1}$ could then be activated by tosylate formation $(40 \%$ yield of product 12) and subsequently be displaced with azide to furnish compound $\mathbf{1 3}$ ( $73 \%$ yield). Finally, the aminomethyl compound 14 was obtained by hydrogenation of azide $\mathbf{1 3}$ (67\% yield), and actually represents the $N$-Boc-imidazoline congener of our initial target compound $\mathbf{1}$. This material $\mathbf{1 4}$ will now be the cornerstone in future efforts for the synthesis of new libraries of chiral polydentate ligands.

In summary, during our studies towards new, optically active tridentate ligands, we considered aminomethyl-substituted pyridyloxazoline $\mathbf{1}$ as a core structure which would be ready for further derivatization at the primary amino function. However, attempts to prepare target compound 1 by reduction of the respective azidomethyl precursor $\mathbf{1 0}$ directly led to the rearranged product $\mathbf{2}$ with a hydroxymethyl-substituted imidazoline ring. This very basic amidine moiety hindered subsequent chemistry and was therefore protected with a tert-butoxycarbonyl group. It was then possible to access the (aminomethyl)(pyridyl)imidazoline $\mathbf{1 4}$ which is now ready for further diversifying derivatization at the primary amino function.

Preparative column chromatography was carried out using Merck silica gel (35-70 $\mu \mathrm{m}$, type $60 \mathrm{~A})$ with hexane, EtOAc and $\mathrm{MeOH}$ as eluents. The column dimensions are given as follows: diameter $x$ height. TLC was performed on Merck aluminum plates coated with silica gel $\mathrm{F}_{254} \cdot{ }^{1} \mathrm{H},{ }^{13} \mathrm{C}$ and ${ }^{15} \mathrm{~N}$ NMR spectra were recorded on a Bruker Avance DRX 500 instrument. Multiplicities of carbon signals were determined with DEPT experiments. MS and HRMS spectra were obtained with a Waters Q-Tof Premier (ESI) spectrometer. IR spectra were recorded on a Bruker Tensor 27 spectrometer equipped with a Golden Gate diamond ATR unit. Elemental analyses were determined with a Euro EA-CHNS instrument from HEKAtech. Optical rotations were measured with a Polartronic M polarimeter from Schmidt and Haensch. Methyl L-serinate hydro- 
chloride was prepared according to a literature procedure. ${ }^{10}$ All other starting materials were commercially available.

\section{Methyl (S)- $N$-(2-Pyridylcarbonyl)serinate (4)}

$N$-Methylmorpholine $(10.3 \mathrm{~mL}, 9.46 \mathrm{~g}, 93.5 \mathrm{mmol})$ and ethyl chloroformate $(4.20 \mathrm{~mL}, 4.85 \mathrm{~g}, 44.7 \mathrm{mmol})$ were added to an icecooled suspension of picolinic acid (3) $(5.00 \mathrm{~g}, 40.6 \mathrm{mmol})$ in anhydrous THF $(50 \mathrm{~mL})$ and the mixture was stirred for $0.5 \mathrm{~h}$ at this temperature. Methyl L-serinate hydrochloride $(7.00 \mathrm{~g}, 44.7 \mathrm{mmol})$ was added and, after further stirring for $2 \mathrm{~h}$ at r.t., the solvent was removed under reduced pressure. The residue was partitioned between EtOAc $(50 \mathrm{~mL})$ and $\mathrm{H}_{2} \mathrm{O}(50 \mathrm{~mL})$, and the aqueous layer was extracted with EtOAc $(2 \times 50 \mathrm{~mL})$. The combined organic layers were dried $\left(\mathrm{MgSO}_{4}\right)$ and filtered, and the solvent was removed under reduced pressure. The residue was purified by column chromatography (silica gel, $7 \mathrm{~cm} \times 11 \mathrm{~cm}$; EtOAc-hexane, $2: 1, R_{f}=0.25$ ) to yield compound $4(6.46 \mathrm{~g}, 28.8 \mathrm{mmol}, 71 \%)$ as a colorless liquid. All spectroscopic data were in accordance with the literature. ${ }^{11}$

$[\alpha]_{\mathrm{D}}{ }^{20}+38.9\left(c 1.03, \mathrm{CH}_{2} \mathrm{Cl}_{2}\right)\left[\right.$ Lit. $\left.^{11}[\alpha]_{\mathrm{D}}{ }^{21}+39.4\left(c 2.5, \mathrm{CHCl}_{3}\right)\right]$.

${ }^{1} \mathrm{H}$ NMR $\left(500 \mathrm{MHz}, \mathrm{CDCl}_{3}\right): \delta=2.87(\mathrm{t}, J=5.7 \mathrm{~Hz}, 1 \mathrm{H}, \mathrm{OH}), 3.82$ $(\mathrm{s}, 3 \mathrm{H}), 4.03-4.14(\mathrm{~m}, 2 \mathrm{H}), 4.87(\mathrm{dt}, J=7.7,3.9 \mathrm{~Hz}, 1 \mathrm{H}), 7.44$ (ddd, $J=7.6,4.8,1.0 \mathrm{~Hz}, 1 \mathrm{H}), 7.84(\mathrm{td}, J=7.7,1.7 \mathrm{~Hz}, 1 \mathrm{H}), 8.16$ (dt, $J=7.9,1.0 \mathrm{~Hz}, 1 \mathrm{H}), 8.58(\mathrm{ddd}, J=4.8,1.5,0.9 \mathrm{~Hz}, 1 \mathrm{H}), 8.80$ $8.85(\mathrm{~m}, 1 \mathrm{H}, \mathrm{NH})$.

${ }^{13} \mathrm{C}\left\{{ }^{1} \mathrm{H}\right\} \mathrm{NMR}\left(125 \mathrm{MHz}, \mathrm{CDCl}_{3}\right): \delta=52.8(\mathrm{CH}), 54.9\left(\mathrm{CH}_{3}\right), 63.5$ $\left(\mathrm{CH}_{2}\right), 122.4(\mathrm{CH}), 126.5(\mathrm{CH}), 137.3(\mathrm{CH}), 148.3(\mathrm{CH}), 149.1(\mathrm{C})$, 164.7 (C), 170.7 (C).

\section{Methyl (S)-O-(tert-Butyldimethylsilyl)- $N$-(2-pyridylcarbon-} yl)serinate (5)

TBSCl (4.81 g, $31.9 \mathrm{mmol})$ and imidazole $(5.00 \mathrm{~g}, 73.5 \mathrm{mmol})$ were added to a solution of compound $4(5.49 \mathrm{~g}, 24.5 \mathrm{mmol})$ in $\mathrm{CH}_{2} \mathrm{Cl}_{2}$ $(70 \mathrm{~mL})$, and the mixture was stirred for $1 \mathrm{~h}$ at r.t. and then filtered. The solvent was removed under reduced pressure and the residue was purified by column chromatography (silica gel, $7 \mathrm{~cm} \times 11 \mathrm{~cm}$; EtOAc-hexane, 1:2, $\left.R_{f}=0.40\right)$ to yield compound $5(7.66 \mathrm{~g}, 22.6$ mmol, $92 \%)$ as a colorless liquid.

$[\alpha]_{\mathrm{D}}^{20}+35.3\left(c 1.13, \mathrm{CH}_{2} \mathrm{Cl}_{2}\right)$.

IR (ATR): 3396 (w), 2953 (w), 2930 (w), 2885 (w), 2857 (w), 1749 (m), $1680(\mathrm{~m}), 1592(\mathrm{w}), 1571(\mathrm{w}), 1512(\mathrm{~s}), 1465(\mathrm{~m}), 1435$ (m), $1380(\mathrm{w}), 1352(\mathrm{~m}), 1292(\mathrm{w}), 1253(\mathrm{~m}), 1207$ (m), $1168(\mathrm{~m}), 1107$ (s), $1043(\mathrm{~m}), 998(\mathrm{~m}), 965(\mathrm{w}), 939(\mathrm{w}) \mathrm{cm}^{-1}$.

${ }^{1} \mathrm{H}$ NMR $\left(500 \mathrm{MHz}, \mathrm{CDCl}_{3}\right): \delta=0.009$ (s, $\left.3 \mathrm{H}\right), 0.011$ (s, $\left.3 \mathrm{H}\right), 0.89$ $(\mathrm{s}, 9 \mathrm{H}), 3.75(\mathrm{~s}, 3 \mathrm{H}), 3.95(\mathrm{dd}, J=10.1,3.5 \mathrm{~Hz}, 1 \mathrm{H}), 4.19$ (dd, $J=$ $10.1,3.0 \mathrm{~Hz}, 1 \mathrm{H}), 4.86(\mathrm{dt}, J=8.6,3.2 \mathrm{~Hz}, 1 \mathrm{H}), 7.43$ (ddd, $J=7.6$, 4.7, $1.2 \mathrm{~Hz}, 1 \mathrm{H}), 7.84(\mathrm{td}, J=7.7,1.7 \mathrm{~Hz}, 1 \mathrm{H}), 8.18(\mathrm{dt}, J=7.8$, $1.1 \mathrm{~Hz}, 1 \mathrm{H}), 8.60(\mathrm{ddd}, J=4.8,1.6,0.8 \mathrm{~Hz}, 1 \mathrm{H}), 8.74-8.78(\mathrm{~m}, 1$ $\mathrm{H})$.

${ }^{13} \mathrm{C}\left\{{ }^{1} \mathrm{H}\right\} \operatorname{NMR}\left(125 \mathrm{MHz}, \mathrm{CDCl}_{3}\right): \delta=-5.6\left(\mathrm{CH}_{3}\right),-5.5\left(\mathrm{CH}_{3}\right), 18.3$ (C), $25.8\left(3 \mathrm{CH}_{3}\right), 52.5(\mathrm{CH}), 54.6\left(\mathrm{CH}_{3}\right), 63.8\left(\mathrm{CH}_{2}\right), 122.4(\mathrm{CH})$, $126.4(\mathrm{CH}), 137.3(\mathrm{CH}), 148.5(\mathrm{CH}), 149.7(\mathrm{C}), 164.3(\mathrm{C}), 170.9$ (C).

HRMS (ESI, positive mode): $m / z\left[\mathrm{M}+\mathrm{Na}^{+}\right]$calcd for $\mathrm{C}_{16} \mathrm{H}_{26} \mathrm{~N}_{2} \mathrm{NaO}_{4} \mathrm{Si}$ : 361.1560; found: 361.1552.

Anal. Calcd for $\mathrm{C}_{16} \mathrm{H}_{26} \mathrm{~N}_{2} \mathrm{O}_{4} \mathrm{Si}$ (338.48): C, 56.78; H, 7.74; N, 8.28. Found: C, 56.77; H, 7.82; N, 8.40.

\section{$N$-[(R)-2-(tert-Butyldimethylsilyloxy)-1-(hydroxymethyl)eth-} yl]picolinamide (8)

Compound $\mathbf{5}(9.48 \mathrm{~g}, 28.0 \mathrm{mmol})$ was added dropwise to an icecooled solution of $\mathrm{LiBH}_{4}(18.3 \mathrm{~mL}$ of a $2 \mathrm{M}$ solution in THF, 36.6 mmol; diluted with $18.3 \mathrm{~mL}$ anhydrous THF) and the mixture was stirred for $3 \mathrm{~h}$ at this temperature. Subsequently, $\mathrm{H}_{2} \mathrm{O}(10 \mathrm{~mL})$ and $10 \% \mathrm{HCl}$ (ca. $20 \mathrm{~mL}$ ) were added with caution. After gas evolution had stopped, sat. aq $\mathrm{NaHCO}_{3}$ solution (ca. $40 \mathrm{~mL}$ ) was added and the mixture was extracted with EtOAc $(4 \times 40 \mathrm{~mL})$. The combined organic layers were dried $\left(\mathrm{MgSO}_{4}\right)$ and filtered, and the solvent was removed under reduced pressure. The residue was purified by column chromatography (silica gel, $7 \mathrm{~cm} \times 12 \mathrm{~cm}$; EtOAc-hexane, 1:1, $\left.R_{f}=0.20\right)$ to yield compound $8(6.88 \mathrm{~g}, 22.2 \mathrm{mmol}, 79 \%)$ as a colorless liquid.

$[\alpha]_{\mathrm{D}}^{20}+3.33\left(c 1.00, \mathrm{CH}_{2} \mathrm{Cl}_{2}\right)$.

IR (ATR): 3384 (m), 2955 (m), 2931 (m), 2885 (w), 2859 (m), 1666 (s), $1593(\mathrm{w}), 1572(\mathrm{w}), 1521(\mathrm{~s}), 1466(\mathrm{~m}), 1436(\mathrm{~m}), 1391(\mathrm{w})$, 1363 (w), 1293 (w), 1255 (m), 1091 (s), 1046 (m), 1022 (m), 1000 (m), $940(\mathrm{w}), 835$ (s), 779 (s) $\mathrm{cm}^{-1}$.

${ }^{1} \mathrm{H}$ NMR $\left(500 \mathrm{MHz}, \mathrm{CDCl}_{3}\right): \delta=0.08(\mathrm{~s}, 3 \mathrm{H}), 0.09$ (s, $\left.3 \mathrm{H}\right), 0.92$ $(\mathrm{s}, 9 \mathrm{H}), 3.01(\mathrm{dd}, J=7.8,4.0 \mathrm{~Hz}, 1 \mathrm{H}), 3.80-3.85(\mathrm{~m}, 1 \mathrm{H}), 3.86-$ $3.98(\mathrm{~m}, 3 \mathrm{H}), 4.13-4.20(\mathrm{~m}, 1 \mathrm{H}), 7.42$ (ddd, $J=7.7,4.7,1.2 \mathrm{~Hz}, 1$ H), $7.85(\mathrm{td}, J=7.7,1.6 \mathrm{~Hz}, 1 \mathrm{H}), 8.19(\mathrm{dt}, J=7.8,1.0 \mathrm{~Hz}, 1 \mathrm{H})$, 8.56 (ddd, $J=4.8,1.6,0.8 \mathrm{~Hz}, 1 \mathrm{H}), 8.63-8.67(\mathrm{~m}, 1 \mathrm{H})$.

${ }^{13} \mathrm{C}\left\{{ }^{1} \mathrm{H}\right\}$ NMR $\left(125 \mathrm{MHz}, \mathrm{CDCl}_{3}\right): \delta=-5.6\left(\mathrm{CH}_{3}\right),-5.4\left(\mathrm{CH}_{3}\right), 18.3$ (C), $26.0\left(3 \mathrm{CH}_{3}\right), 52.5(\mathrm{CH}), 63.8\left(\mathrm{CH}_{2}\right), 64.2\left(\mathrm{CH}_{2}\right), 122.3(\mathrm{CH})$, $126.3(\mathrm{CH}), 137.4(\mathrm{CH}), 148.4(\mathrm{CH}), 150.0(\mathrm{C}), 164.9(\mathrm{C})$.

HRMS (ESI, positive mode): $m / z\left[\mathrm{M}+\mathrm{Na}^{+}\right]$calcd for $\mathrm{C}_{15} \mathrm{H}_{26} \mathrm{~N}_{2} \mathrm{NaO}_{3} \mathrm{Si}$ : 333.1610; found: 333.1612.

Anal. Calcd for $\mathrm{C}_{15} \mathrm{H}_{26} \mathrm{~N}_{2} \mathrm{O}_{3} \mathrm{Si}$ (310.47): C, 58.03; H, 8.44; N, 9.02. Found: C, 58.01; H, 8.44; N, 9.02.

\section{(R)-4-(tert-Butyldimethylsilyloxymethyl)-2-(2-pyridyl)-4,5-di-} hydrooxazole (6)

TsCl (1.47 g, $7.73 \mathrm{mmol})$, DMAP (78 $\mathrm{mg}, 0.64 \mathrm{mmol})$ and $\mathrm{Et}_{3} \mathrm{~N}$ $(4.48 \mathrm{~mL}, 3.26 \mathrm{~g}, 32.2 \mathrm{mmol})$ were added to a solution of compound $8(2.00 \mathrm{~g}, 6.44 \mathrm{mmol})$ in DCE $(40 \mathrm{~mL})$ and the resulting mixture was heated to reflux for $16 \mathrm{~h}$. The organic layer was washed with $\mathrm{H}_{2} \mathrm{O}(3 \times 30 \mathrm{~mL})$, dried $\left(\mathrm{MgSO}_{4}\right)$ and filtered, and the solvent was removed under reduced pressure. The residue was purified by column chromatography (silica gel, $5 \mathrm{~cm} \times 12 \mathrm{~cm}$; EtOAc-hexane, 1:1, $\left.R_{f}=0.20\right)$ to yield compound $6(1.45 \mathrm{~g}, 4.96 \mathrm{mmol}, 77 \%)$ as a colorless liquid.

$[\alpha]_{\mathrm{D}}^{20}-37.5\left(c 0.99, \mathrm{CH}_{2} \mathrm{Cl}_{2}\right)$.

IR (ATR): 2955 (m), 2930 (m), 2899 (w), 2858 (m), 1643 (m), 1585 (w), $1572(\mathrm{w}), 1519(\mathrm{w}), 1473(\mathrm{~m}), 1442(\mathrm{~m}), 1363(\mathrm{~m}), 1253(\mathrm{~m})$, $1109(\mathrm{~m}), 1045(\mathrm{~m}), 1009(\mathrm{~m}), 967(\mathrm{~m}), 940(\mathrm{w}), 897(\mathrm{w}), 835(\mathrm{~s})$, $803(\mathrm{~m}), 777(\mathrm{~s}), 746(\mathrm{~m}) \mathrm{cm}^{-1}$.

${ }^{1} \mathrm{H}$ NMR $\left(500 \mathrm{MHz}, \mathrm{CDCl}_{3}\right): \delta=0.04(\mathrm{~s}, 3 \mathrm{H}), 0.07(\mathrm{~s}, 3 \mathrm{H}), 0.86$ (s, $9 \mathrm{H}), 3.65-3.70(\mathrm{~m}, 1 \mathrm{H}), 3.94(\mathrm{dd}, J=10.2,3.4 \mathrm{~Hz}, 1 \mathrm{H}), 4.42$ $4.50(\mathrm{~m}, 2 \mathrm{H}), 4.51-4.57(\mathrm{~m}, 1 \mathrm{H}), 7.38$ (ddd, $J=7.6,4.8,1.1 \mathrm{~Hz}, 1$ H), $7.77(\mathrm{td}, J=7.8,1.6 \mathrm{~Hz}, 1 \mathrm{H}), 8.03(\mathrm{dt}, J=7.9,1.0 \mathrm{~Hz}, 1 \mathrm{H})$, 8.70 (ddd, $J=4.8,1.7,0.9 \mathrm{~Hz}, 1 \mathrm{H})$.

${ }^{13} \mathrm{C}\left\{{ }^{1} \mathrm{H}\right\}$ NMR $\left(125 \mathrm{MHz}, \mathrm{CDCl}_{3}\right): \delta=-5.2\left(2 \mathrm{CH}_{3}\right), 18.4(\mathrm{C}), 26.0$ $\left(3 \mathrm{CH}_{3}\right), 65.2\left(\mathrm{CH}_{2}\right), 68.7(\mathrm{CH}), 71.2\left(\mathrm{CH}_{2}\right), 124.0(\mathrm{CH}), 125.7$ $(\mathrm{CH}), 136.7(\mathrm{CH}), 147.0(\mathrm{C}), 149.9(\mathrm{CH}), 164.1(\mathrm{C})$.

HRMS (ESI, positive mode): $m / z\left[\mathrm{M}+\mathrm{Na}^{+}\right]$calcd for $\mathrm{C}_{15} \mathrm{H}_{24} \mathrm{~N}_{2} \mathrm{NaO}_{2} \mathrm{Si}$ : 315.1505 ; found: 315.1500 .

Anal. Calcd for $\mathrm{C}_{15} \mathrm{H}_{24} \mathrm{~N}_{2} \mathrm{O}_{2} \mathrm{Si}$ (292.45): C, 61.60; H, 8.27; N, 9.58. Found: C, 61.59; H, 8.29; N, 9.52.

(S)-4-(Hydroxymethyl)-2-(2-pyridyl)-4,5-dihydrooxazole (7) TBAF $3 \mathrm{H}_{2} \mathrm{O}(1.94 \mathrm{~g}, 6.16 \mathrm{mmol})$ was added to a solution of compound $6(1.50 \mathrm{~g}, 5.13 \mathrm{mmol})$ in THF $(50 \mathrm{~mL})$ and the resulting mixture was stirred for $1 \mathrm{~h}$ at r.t. The solvent was removed under reduced pressure and the residue was purified by column chromatography (silica gel, $5 \mathrm{~cm} \times 10 \mathrm{~cm}$; EtOAc-MeOH, 5:1, $R_{f}=0.24$ ) to yield compound $7(0.850 \mathrm{~g}, 4.77 \mathrm{mmol}, 93 \%)$ as a colorless oil.

$[\alpha]_{\mathrm{D}}^{20}-61.6(c 0.69, \mathrm{MeOH})$.

IR (ATR): 3288 (m), 2982 (w), 2927 (w), 2874 (w), 1664 (m), 1582 (m), $1483(\mathrm{~m}), 1432(\mathrm{~m}), 1398(\mathrm{w}), 1381(\mathrm{~m}), 1360(\mathrm{~s}), 1343(\mathrm{~m})$, $1301(\mathrm{~m}), 1285(\mathrm{~m}), 1270(\mathrm{~m}), 1247(\mathrm{~m}), 1222(\mathrm{~m}), 1199(\mathrm{w}), 1155$ (w), $1125(\mathrm{~s}), 1098(\mathrm{~m}), 1086(\mathrm{~m}), 1070(\mathrm{~s}), 1042(\mathrm{~m}), 995(\mathrm{~m}), 983$ (m), $952(\mathrm{~s}), 933(\mathrm{~m}), 908(\mathrm{w}), 890(\mathrm{~m}) \mathrm{cm}^{-1}$. 
${ }^{1} \mathrm{H}$ NMR $\left(500 \mathrm{MHz}, \mathrm{CDCl}_{3}\right): \delta=3.18(\mathrm{~s}, 1 \mathrm{H}), 3.70(\mathrm{dd}, J=11.6$, $4.0 \mathrm{~Hz}, 1 \mathrm{H}), 4.01(\mathrm{dd}, J=11.6,3.4 \mathrm{~Hz}, 1 \mathrm{H}), 4.42-4.46(\mathrm{~m}, 1 \mathrm{H})$, $4.47-4.53(\mathrm{~m}, 1 \mathrm{H}), 4.57$ (dd, $J=9.5,7.2 \mathrm{~Hz}, 1 \mathrm{H}), 7.35$ (ddd, $J=$ 7.6, 4.8, 0.9 Hz, $1 \mathrm{H}), 7.73(\mathrm{td}, J=7.7,1.7 \mathrm{~Hz}, 1 \mathrm{H}), 7.93(\mathrm{dt}, J=$ $7.9,1.2 \mathrm{~Hz}, 1 \mathrm{H}), 8.64$ (ddd, $J=4.8,1.6,0.9 \mathrm{~Hz}, 1 \mathrm{H})$.

${ }^{13} \mathrm{C}\left\{{ }^{1} \mathrm{H}\right\}$ NMR $\left(125 \mathrm{MHz}, \mathrm{CDCl}_{3}\right): \delta=64.0\left(\mathrm{CH}_{2}\right), 68.6(\mathrm{CH}), 70.0$ $\left(\mathrm{CH}_{2}\right), 124.0(\mathrm{CH}), 125.9(\mathrm{CH}), 136.8(\mathrm{CH}), 146.2(\mathrm{C}), 149.9(\mathrm{CH})$, $164.5(\mathrm{C})$.

HRMS (ESI, positive mode): $m / z\left[\mathrm{M}+\mathrm{Na}^{+}\right]$calcd for $\mathrm{C}_{9} \mathrm{H}_{10} \mathrm{~N}_{2} \mathrm{NaO}_{2}$ : 201.0640; found: 201.0635 .

\section{(R)-4-[(4-Methylphenylsulfonyloxy)methyl]-2-(2-pyridyl)-4,5-} dihydrooxazole (9)

TsCl $(1.07 \mathrm{~g}, 5.60 \mathrm{mmol})$ was added to a solution of compound 7 $(0.665 \mathrm{~g}, 3.73 \mathrm{mmol})$ in $15 \%$ aq $\mathrm{KOH}$ solution $(25 \mathrm{~mL})$ and $\mathrm{CH}_{2} \mathrm{Cl}_{2}$ $(25 \mathrm{~mL})$, and the resulting mixture was heated to reflux for $3 \mathrm{~h}$. The layers were separated and the aqueous layer was extracted with $\mathrm{CH}_{2} \mathrm{Cl}_{2}(50 \mathrm{~mL})$. The combined organic layers were dried $\left(\mathrm{MgSO}_{4}\right)$ and filtered, and the solvent was removed under reduced pressure. The residue was purified by column chromatography (silica gel, 5 $\mathrm{cm} \times 8 \mathrm{~cm}$; EtOAc-MeOH, 5:1, $R_{f}=0.40$ ) to yield compound 9 $(1.18 \mathrm{~g}, 3.56 \mathrm{mmol}, 95 \%)$ as a colorless solid; $\mathrm{mp} 113{ }^{\circ} \mathrm{C}$.

$[\alpha]_{\mathrm{D}}{ }^{20}-82.9\left(c 1.15, \mathrm{CH}_{2} \mathrm{Cl}_{2}\right)$.

IR (ATR): 3055 (w), 2968 (w), 2902 (w), 2324 (w), 1732 (w), 1645 (m), $1599(\mathrm{~m}), 1568(\mathrm{~m}), 1494(\mathrm{w}), 1471(\mathrm{~m}), 1442(\mathrm{~m}), 1348(\mathrm{~m})$, $1306(\mathrm{~m}), 1292(\mathrm{~m}), 1280(\mathrm{~m}), 1246(\mathrm{~m}), 1213(\mathrm{~m}), 1175(\mathrm{~s}), 1121$ (m), $1098(\mathrm{~m}), 1080(\mathrm{~m}), 1039(\mathrm{~m}), 1012(\mathrm{~m}), 994(\mathrm{~m}), 966(\mathrm{~m}), 948$ (s), $932(\mathrm{~m}), 898(\mathrm{~m}), 866(\mathrm{~m}) \mathrm{cm}^{-1}$

${ }^{1} \mathrm{H}$ NMR $\left(500 \mathrm{MHz}, \mathrm{CDCl}_{3}\right): \delta=2.41(\mathrm{~s}, 3 \mathrm{H}), 4.05(\mathrm{dd}, J=10.0$, $6.6 \mathrm{~Hz}, 1 \mathrm{H}), 4.29(\mathrm{dd}, J=10.0,3.7 \mathrm{~Hz}, 1 \mathrm{H}), 4.36(\mathrm{dd}, J=8.1,6.7$ $\mathrm{Hz}, 1 \mathrm{H}), 4.51-4.62$ (m, $2 \mathrm{H}), 7.30$ (d, $J=8.2 \mathrm{~Hz}, 2 \mathrm{H}), 7.39$ (ddd, $J=7.7,4.7,1.1 \mathrm{~Hz}, 1 \mathrm{H}), 7.72-7.79(\mathrm{~m}, 3 \mathrm{H}), 7.91-7.94(\mathrm{~m}, 1 \mathrm{H})$, $8.68(\mathrm{ddd}, J=4.9,1.8,0.9 \mathrm{~Hz}, 1 \mathrm{H})$.

${ }^{13} \mathrm{C}\left\{{ }^{1} \mathrm{H}\right\}$ NMR $\left(125 \mathrm{MHz}, \mathrm{CDCl}_{3}\right): \delta=21.7\left(\mathrm{CH}_{3}\right), 65.5(\mathrm{CH}), 70.5$ $\left(\mathrm{CH}_{2}\right), 70.6\left(\mathrm{CH}_{2}\right), 124.2(\mathrm{CH}), 126.1(\mathrm{CH}), 128.1(2 \mathrm{CH}), 130.0(2$ $\mathrm{CH}), 132.6(\mathrm{C}), 136.8(\mathrm{CH}), 145.2(\mathrm{C}), 146.1(\mathrm{C}), 150.0(\mathrm{CH})$, $165.0(\mathrm{C})$.

HRMS (ESI, positive mode): $m / z\left[\mathrm{M}+\mathrm{Na}^{+}\right]$calcd for $\mathrm{C}_{16} \mathrm{H}_{16} \mathrm{~N}_{2} \mathrm{NaO}_{4} \mathrm{~S}$ : 355.0728; found: 355.0728 .

Anal. Calcd for $\mathrm{C}_{16} \mathrm{H}_{16} \mathrm{~N}_{2} \mathrm{O}_{4} \mathrm{~S}$ (332.37): C, 57.82; H, 4.85; N, 8.43; S, 9.65. Found: C, 57.91; H, 4.86; N, 8.47; S, 9.65 .

\section{(S)-4-(Azidomethyl)-2-(2-pyridyl)-4,5-dihydrooxazole (10)}

$\mathrm{NaN}_{3}(3.39 \mathrm{~g}, 52.1 \mathrm{mmol})$ was added to a solution of compound 9 $(3.46 \mathrm{~g}, 10.4 \mathrm{mmol})$ in $\mathrm{EtOH}(150 \mathrm{~mL})$ and the resulting mixture was heated to reflux for $16 \mathrm{~h}$. After the solvent was removed, the residue was partitioned between $\mathrm{CH}_{2} \mathrm{Cl}_{2}(100 \mathrm{~mL})$ and $\mathrm{H}_{2} \mathrm{O}(100$ $\mathrm{mL})$, and the aqueous layer was extracted with $\mathrm{CH}_{2} \mathrm{Cl}_{2}(50 \mathrm{~mL})$. The combined organic layers were dried $\left(\mathrm{MgSO}_{4}\right)$ and filtered, and the solvent was removed under reduced pressure. The residue was purified by column chromatography (silica gel, $5 \mathrm{~cm} \times 10 \mathrm{~cm}$; EtOAc-hexane, 9:1, $\left.R_{f}=0.15\right)$ to yield compound $\mathbf{1 0}(2.12 \mathrm{~g}, 10.4$ mmol, $99 \%$ ) as a light yellow oil.

$[\alpha]_{\mathrm{D}}^{20}-112.5\left(c 1.01, \mathrm{CH}_{2} \mathrm{Cl}_{2}\right)$.

IR (ATR): 3057 (w), 2969 (w), 2902 (w), 2096 (vs), 1639 (m), 1581 $(\mathrm{m}), 1569(\mathrm{~m}), 1516(\mathrm{w}), 1469(\mathrm{~m}), 1440(\mathrm{~m}), 1366(\mathrm{~m}), 1258(\mathrm{~m})$ 1151 (w), 1102 (s), $1066(\mathrm{~m}), 1043$ (m), 994 (m), 965 (m), 931 (m), $905(\mathrm{~m}), 856(\mathrm{w}), 800(\mathrm{~m}) \mathrm{cm}^{-1}$.

${ }^{1} \mathrm{H}$ NMR $\left(500 \mathrm{MHz}, \mathrm{CDCl}_{3}\right): \delta=3.53-3.62(\mathrm{~m}, 2 \mathrm{H}), 4.33-4.41(\mathrm{~m}$, $1 \mathrm{H}), 4.54-4.62$ (m, $2 \mathrm{H}), 7.42$ (ddd, $J=7.6,4.8,1.0 \mathrm{~Hz}, 1 \mathrm{H}), 7.80$ $(\mathrm{td}, J=7.8,1.7 \mathrm{~Hz}, 1 \mathrm{H}), 8.05(\mathrm{dt}, J=7.9,1.0 \mathrm{~Hz}, 1 \mathrm{H}), 8.72$ (ddd, $J=4.8,1.6,1.0 \mathrm{~Hz}, 1 \mathrm{H})$.

${ }^{13} \mathrm{C}\left\{{ }^{1} \mathrm{H}\right\}$ NMR $\left(125 \mathrm{MHz}, \mathrm{CDCl}_{3}\right): \delta=54.2\left(\mathrm{CH}_{2}\right), 66.5(\mathrm{CH}), 70.7$ $\left(\mathrm{CH}_{2}\right), 124.2(\mathrm{CH}), 125.9(\mathrm{CH}), 136.7(\mathrm{CH}), 146.2(\mathrm{C}), 149.9(\mathrm{CH})$, $164.5(\mathrm{C})$.
HRMS (ESI, positive mode): $m / z\left[\mathrm{M}+\mathrm{Na}^{+}\right]$calcd for $\mathrm{C}_{9} \mathrm{H}_{9} \mathrm{~N}_{5} \mathrm{NaO}$ : 226.0705; found: 226.0699 .

Anal. Calcd for $\mathrm{C}_{9} \mathrm{H}_{9} \mathrm{~N}_{5} \mathrm{O}$ (203.21): C, 53.20; H, 4.46; N, 34.37 . Found: C, 53.16; H, 4.47; N, 34.29 .

\section{(S)-4-(Hydroxymethyl)-2-(2-pyridyl)-4,5-dihydro-1 $H$-imidaz-} ole (2)

A suspension of compound $\mathbf{1 0}(2.21 \mathrm{~g}, 10.9 \mathrm{mmol})$ and $\mathrm{Pd} / \mathrm{C}(221$ $\mathrm{mg}, 10 \% \mathrm{w} / \mathrm{w} \mathrm{Pd})$ in $\mathrm{MeOH}(50 \mathrm{~mL})$ was degassed (three cycles of freeze, pump and thaw) and then stirred under an atmosphere of $\mathrm{H}_{2}$ (1 atm) for $16 \mathrm{~h}$ at $23{ }^{\circ} \mathrm{C}$. The mixture was filtered, then the solvent was removed under reduced pressure to yield compound $2(1.82 \mathrm{~g}$, $10.3 \mathrm{mmol}, 95 \%$ ) as a yellow oil.

$[\alpha]_{\mathrm{D}}{ }^{20}+55.3(c 1.11, \mathrm{MeOH})$

IR (ATR): 3279 (br), 2927 (m), 2862 (m), 2453 (w), 1659 (w), 1601 (m), $1566(\mathrm{~m}), 1487(\mathrm{~m}), 1456(\mathrm{~m}), 1421(\mathrm{~m}), 1331(\mathrm{~m}), 1271(\mathrm{~m})$, $1189(\mathrm{~m}), 1096(\mathrm{~m}), 1045(\mathrm{~m}), 979(\mathrm{~m}), 801(\mathrm{~m}) \mathrm{cm}^{-1}$.

${ }^{1} \mathrm{H}$ NMR $\left(500 \mathrm{MHz}, \mathrm{CD}_{3} \mathrm{OD}\right): \delta=3.61(\mathrm{dd}, J=11.0,5.6 \mathrm{~Hz}, 1 \mathrm{H})$, $3.65(\mathrm{dd}, J=11.1,5.5 \mathrm{~Hz}, 1 \mathrm{H}), 3.70(\mathrm{dd}, J=12.3,7.5 \mathrm{~Hz}, 1 \mathrm{H})$, 3.92 (dd, $J=12.2,10.9 \mathrm{~Hz}, 1 \mathrm{H}), 4.19$ (ddt, $J=10.7,7.5,5.6 \mathrm{~Hz}, 1$ H), 7.50 (ddd, $J=7.5,4.9,1.0 \mathrm{~Hz}, 1 \mathrm{H}), 7.90(\mathrm{td}, J=7.8,1.7 \mathrm{~Hz}, 1$ H), $8.02(\mathrm{dt}, J=7.9,1.0 \mathrm{~Hz}, 1 \mathrm{H}), 8.63(\mathrm{ddd}, J=4.9,1.5,1.1 \mathrm{~Hz}, 1$ $\mathrm{H})$.

${ }^{13} \mathrm{C}\left\{{ }^{1} \mathrm{H}\right\} \mathrm{NMR}\left(125 \mathrm{MHz}, \mathrm{CD}_{3} \mathrm{OD}\right): \delta=53.5\left(\mathrm{CH}_{2}\right), 63.6(\mathrm{CH}), 65.5$ $\left(\mathrm{CH}_{2}\right), 123.5(\mathrm{CH}), 126.9(\mathrm{CH}), 138.2(\mathrm{CH}), 148.8(\mathrm{C}), 150.2(\mathrm{CH})$, $165.5(\mathrm{C})$.

HRMS (ESI, positive mode): $m / z\left[\mathrm{M}+\mathrm{H}^{+}\right]$calcd for $\mathrm{C}_{9} \mathrm{H}_{12} \mathrm{~N}_{3} \mathrm{O}$ : 178.0980; found: 178.0975 .

Anal. Calcd for $\mathrm{C}_{9} \mathrm{H}_{11} \mathrm{~N}_{3} \mathrm{O}$ (177.21): C, 61.00; H, 6.26; N, 23.71 . Found: C, 60.89; H, 6.57; N, 23.73.

(S)-1-(tert-Butoxycarbonyl)-4-(hydroxymethyl)-2-(2-pyridyl)4,5-dihydro- $1 \mathrm{H}$-imidazole (11)

$\mathrm{K}_{2} \mathrm{CO}_{3}(2.70 \mathrm{~g}, 19.5 \mathrm{mmol})$ and $\mathrm{Boc}_{2} \mathrm{O}(5.33 \mathrm{~g}, 24.4 \mathrm{mmol})$ were added to a solution of compound $2(1.73 \mathrm{~g}, 9.76 \mathrm{mmol})$ in $\mathrm{H}_{2} \mathrm{O}_{-}$ THF (30 mL, 1:3) and the resulting mixture was heated to reflux for $24 \mathrm{~h}$. All volatile materials were then removed under reduced pressure, the residue was partitioned between $\mathrm{CH}_{2} \mathrm{Cl}_{2}(100 \mathrm{~mL})$ and $\mathrm{H}_{2} \mathrm{O}(100 \mathrm{~mL})$, the aqueous layer was extracted with $\mathrm{CH}_{2} \mathrm{Cl}_{2}(50$ $\mathrm{mL}$ ), and the combined organic layers were dried $\left(\mathrm{MgSO}_{4}\right)$ and filtered. The solvent was removed under reduced pressure to yield compound 11 as a mixture with $\mathrm{Boc}_{2} \mathrm{O}(2.30 \mathrm{~g})$ as crude material $\left(\mathrm{Boc}_{2} \mathrm{O} / \mathbf{1 1}, 1: 3\right.$ by ${ }^{1} \mathrm{H}$ NMR spectroscopy; i.e., $1.82 \mathrm{~g}, 6.57 \mathrm{mmol}$, $67 \%$ ), which was used for the next step without further purification. An analytically pure sample was obtained by column chromatography (silica gel, $3 \mathrm{~cm} \times 10 \mathrm{~cm}$; EtOAc-MeOH, 5:1, $R_{f}=0.22$ ) as a light yellow oil.

$[\alpha]_{\mathrm{D}}^{20}+92.5\left(c 0.46, \mathrm{CH}_{2} \mathrm{Cl}_{2}\right)$.

IR (ATR): 3312 (br), 2979 (w), 2932 (w), 2871 (w), 1708 (s), 1631 (m), $1588(\mathrm{w}), 1476(\mathrm{w}), 1365(\mathrm{vs}), 1286(\mathrm{~m}), 1138(\mathrm{vs}), 1049(\mathrm{w})$, $1025(\mathrm{w}), 917(\mathrm{w}), 729(\mathrm{~s}) \mathrm{cm}^{-1}$.

${ }^{1} \mathrm{H} \mathrm{NMR}\left(500 \mathrm{MHz}, \mathrm{CDCl}_{3}\right): \delta=1.21\left(\mathrm{~s}, 9 \mathrm{H}, 3 \mathrm{CH}_{3}\right), 3.64(\mathrm{dd}, J=$ $11.4,5.0 \mathrm{~Hz}, 1 \mathrm{H}, 4-\mathrm{CHH}), 3.74(\mathrm{dd}, J=11.4,4.5 \mathrm{~Hz}, 1 \mathrm{H}, 4-\mathrm{CH} H)$, 3.84 (dd, $J=10.5,8.0 \mathrm{~Hz}, 1 \mathrm{H}, 5-\mathrm{H}), 3.99(\mathrm{t}, J=10.4 \mathrm{~Hz}, 1 \mathrm{H}, 5-$ H), 4.28 (ddt, $J=10.3,8.1,4.7 \mathrm{~Hz}, 1 \mathrm{H}, 4-\mathrm{H}), 7.29$ (ddd, $J=7.7$, 4.9, $\left.1.1 \mathrm{~Hz}, 1 \mathrm{H}, 5^{\prime}-\mathrm{H}\right), 7.47-7.50$ (m, $\left.1 \mathrm{H}, 3^{\prime}-\mathrm{H}\right), 7.70$ (td, $J=7.7$, $\left.1.7 \mathrm{~Hz}, 1 \mathrm{H}, 4^{\prime}-\mathrm{H}\right), 8.58$ (ddd, $J=4.9,1.7,1.1 \mathrm{~Hz}, 1 \mathrm{H}, 6^{\prime}-\mathrm{H}$ ).

${ }^{13} \mathrm{C}\left\{{ }^{1} \mathrm{H}\right\}$ NMR $\left(125 \mathrm{MHz}, \mathrm{CDCl}_{3}\right): \delta=27.8\left(3 \mathrm{CH}_{3}, \mathrm{C}\left(\mathrm{CH}_{3}\right)_{3}\right), 49.3$ $\left(\mathrm{CH}_{2}, \mathrm{C}-5\right), 64.3\left(\mathrm{CH}_{2}, 4-\mathrm{CH}_{2}\right), 66.8(\mathrm{CH}, \mathrm{C}-4), 81.9\left(\mathrm{C}, \mathrm{C}\left(\mathrm{CH}_{3}\right)_{3}\right)$, $123.4\left(\mathrm{CH}, \mathrm{C}-3^{\prime}\right), 124.3\left(\mathrm{CH}, \mathrm{C}-5^{\prime}\right), 136.3\left(\mathrm{CH}, \mathrm{C}-4^{\prime}\right), 148.8(\mathrm{CH}$, C-6'), $150.4(\mathrm{C}, \mathrm{C}=\mathrm{O}), 151.4\left(\mathrm{C}, \mathrm{C}-2^{\prime}\right), 159.4(\mathrm{C}, \mathrm{C}-2)$.

${ }^{15} \mathrm{~N}$ NMR (HMBC, $\left.50.7 \mathrm{MHz}, \mathrm{CDCl}_{3}\right): \delta=-74.1\left(\mathrm{~N}-1^{\prime}\right),-122.8(\mathrm{~N}-$ $3),-244.5(\mathrm{~N}-1)$.

HRMS (ESI, positive mode): $m / z\left[\mathrm{M}+\mathrm{Na}^{+}\right]$calcd for $\mathrm{C}_{14} \mathrm{H}_{19} \mathrm{~N}_{3} \mathrm{NaO}_{3}$ : 300.1324; found: 300.1319 . 
(S)-1-(tert-Butoxycarbonyl)-4-[(4-methylphenylsulfonyloxy)methyl]-2-(2-pyridyl)-4,5-dihydro-1H-imidazole (12) $\mathrm{TsCl}(7.23 \mathrm{~g}, 37.9 \mathrm{mmol})$ was added to a solution of crude compound 11 (2.10 g, $\mathrm{Boc}_{2} \mathrm{O} / \mathbf{1 1}, 1: 3$ by ${ }^{1} \mathrm{H}$ NMR spectroscopy; i.e., $1.66 \mathrm{~g}, 6.00 \mathrm{mmol})$ in $15 \%$ aq $\mathrm{KOH}$ solution $(50 \mathrm{~mL})$ and $\mathrm{CH}_{2} \mathrm{Cl}_{2}$ $(50 \mathrm{~mL})$, and the resulting mixture was heated to reflux for $3 \mathrm{~h}$. The layers were separated and the aqueous layer was extracted with $\mathrm{CH}_{2} \mathrm{Cl}_{2}(50 \mathrm{~mL})$. The combined organic layers were dried $\left(\mathrm{MgSO}_{4}\right)$ and filtered, and the solvent was removed under reduced pressure. The residue was purified by column chromatography (silica gel, 5 $\mathrm{cm} \times 10 \mathrm{~cm}$; EtOAc-MeOH, 5:1, $R_{f}=0.23$ ) to yield compound 12 (1.03 g, $2.39 \mathrm{mmol}, 40 \% ; 27 \%$ over two steps) as a yellow oil.

$[\alpha]_{\mathrm{D}}^{20}+65.8\left(c 0.25, \mathrm{CH}_{2} \mathrm{Cl}_{2}\right)$.

IR (ATR): 2978 (w), 2931 (w), 2871 (w), 1708 (s), 1630 (w), 1588 (w), $1474(\mathrm{w}), 1365(\mathrm{vs}), 1288(\mathrm{~m}), 1175(\mathrm{vs}), 1096(\mathrm{~m}), 1049(\mathrm{w})$, $982(\mathrm{~s}), 950(\mathrm{~m}), 815(\mathrm{w}), 790(\mathrm{~m}), 665(\mathrm{~m}), 554(\mathrm{~s}) \mathrm{cm}^{-1}$.

${ }^{1} \mathrm{H}$ NMR $\left(500 \mathrm{MHz}, \mathrm{CDCl}_{3}\right): \delta=1.21(\mathrm{~s}, 9 \mathrm{H}), 2.43(\mathrm{~s}, 3 \mathrm{H}), 3.81$ (dd, $J=11.1,7.2 \mathrm{~Hz}, 1 \mathrm{H}), 3.99-4.13(\mathrm{~m}, 2 \mathrm{H}), 4.27$ (dd, $J=9.9$, $4.1 \mathrm{~Hz}, 1 \mathrm{H}), 4.36-4.48(\mathrm{~m}, 1 \mathrm{H}), 7.29-7.37(\mathrm{~m}, 3 \mathrm{H}), 7.46(\mathrm{dt}, J=$ $8.0,0.9 \mathrm{~Hz}, 1 \mathrm{H}), 7.72(\mathrm{td}, J=7.8,1.8 \mathrm{~Hz}, 1 \mathrm{H}), 7.77-7.81(\mathrm{~m}, 2 \mathrm{H})$, 8.61 (ddd, $J=4.9,1.5,1.1 \mathrm{~Hz}, 1 \mathrm{H})$.

${ }^{13} \mathrm{C}\left\{{ }^{1} \mathrm{H}\right\}$ NMR $\left(125 \mathrm{MHz}, \mathrm{CDCl}_{3}\right): \delta=21.8\left(\mathrm{CH}_{3}\right), 27.8\left(3 \mathrm{CH}_{3}\right)$, $50.0\left(\mathrm{CH}_{2}\right), 63.5(\mathrm{CH}), 70.9\left(\mathrm{CH}_{2}\right), 82.4(\mathrm{C}), 123.4(\mathrm{CH}), 124.5$ $(\mathrm{CH}), 128.2(2 \mathrm{CH}), 130.1(2 \mathrm{CH}), 132.6(\mathrm{C}), 136.4(\mathrm{CH}), 145.1$ (C), 149.0 (CH), 150.2 (C), $151.1(\mathrm{C}), 160.5(\mathrm{C})$.

HRMS (ESI, positive mode): $m / z\left[\mathrm{M}+\mathrm{Na}^{+}\right]$calcd for $\mathrm{C}_{21} \mathrm{H}_{25} \mathrm{~N}_{3} \mathrm{NaO}_{5} \mathrm{~S}: 454.1413$; found: 454.1403.

(S)-4-(Azidomethyl)-1-(tert-butoxycarbonyl)-2-(2-pyridyl)-4,5dihydro-1 $H$-imidazole (13)

A solution of $\mathrm{NaN}_{3}(776 \mathrm{mg}, 11.9 \mathrm{mmol})$ and compound $12(1.03 \mathrm{~g}$, $2.39 \mathrm{mmol})$ in $\mathrm{EtOH}(20 \mathrm{~mL})$ was heated to reflux for $16 \mathrm{~h}$. Then, the solvent was removed under reduced pressure and the residue was partitioned between $\mathrm{CH}_{2} \mathrm{Cl}_{2}(25 \mathrm{~mL})$ and $\mathrm{H}_{2} \mathrm{O}(25 \mathrm{~mL})$. The aqueous layer was extracted with $\mathrm{CH}_{2} \mathrm{Cl}_{2}(25 \mathrm{~mL})$, the combined organic layers were dried $\left(\mathrm{MgSO}_{4}\right)$ and filtered, and the solvent was removed under reduced pressure to yield compound $13(530 \mathrm{mg}$, $1.75 \mathrm{mmol}, 73 \%$ ) as a yellow oil.

$[\alpha]_{\mathrm{D}}^{20}+95.6\left(c 0.79, \mathrm{CH}_{2} \mathrm{Cl}_{2}\right)$.

IR (ATR): 2979 (w), 2932 (w), 2102 (s), 1711 (s), 1633 (w), 1587 (w), $1520(\mathrm{w}), 1472(\mathrm{w}), 1368(\mathrm{vs}), 1277(\mathrm{~m}), 1259(\mathrm{~m}), 1168(\mathrm{~m})$, $1142(\mathrm{~s}), 997(\mathrm{w}), 982(\mathrm{~s}) \mathrm{cm}^{-1}$.

${ }^{1} \mathrm{H}$ NMR $\left(500 \mathrm{MHz}, \mathrm{CDCl}_{3}\right): \delta=1.20(\mathrm{~s}, 9 \mathrm{H}), 3.51$ (dd, $J=12.4$, $4.7 \mathrm{~Hz}, 1 \mathrm{H}), 3.59$ (dd, $J=12.4,5.4 \mathrm{~Hz}, 1 \mathrm{H}), 3.82$ (dd, $J=10.9,7.1$ $\mathrm{Hz}, 1 \mathrm{H}), 4.07(\mathrm{t}, J=10.6 \mathrm{~Hz}, 1 \mathrm{H}), 4.36-4.42(\mathrm{~m}, 1 \mathrm{H}), 7.33$ (ddd, $J=7.7,4.8,1.1 \mathrm{~Hz}, 1 \mathrm{H}), 7.53(\mathrm{dt}, J=7.7,1.0 \mathrm{~Hz}, 1 \mathrm{H}), 7.74(\mathrm{td}$, $J=7.7,1.5 \mathrm{~Hz}, 1 \mathrm{H}), 8.62(\mathrm{ddd}, J=5.4,1.5,1.0 \mathrm{~Hz}, 1 \mathrm{H})$.

${ }^{13} \mathrm{C}\left\{{ }^{1} \mathrm{H}\right\}$ NMR $\left(125 \mathrm{MHz}, \mathrm{CDCl}_{3}\right): \delta=27.7\left(3 \mathrm{CH}_{3}\right), 50.2\left(\mathrm{CH}_{2}\right)$, $54.5\left(\mathrm{CH}_{2}\right), 64.6(\mathrm{CH}), 82.1(\mathrm{C}), 123.2(\mathrm{CH}), 124.3(\mathrm{CH}), 136.3$ (CH), 148.9 (CH), $150.3(\mathrm{C}), 151.4(\mathrm{C}), 159.9(\mathrm{C})$.

HRMS (ESI, positive mode): $m / z\left[\mathrm{M}+\mathrm{Na}^{+}\right]$calcd for $\mathrm{C}_{14} \mathrm{H}_{18} \mathrm{~N}_{6} \mathrm{NaO}_{2}$ : 325.1389; found: 325.1380 .

\section{(R)-4-(Aminomethyl)-1-(tert-butoxycarbonyl)-2-(2-pyridyl)-} 4,5-dihydro-1 $H$-imidazole (14)

A suspension of compound $\mathbf{1 3}(208 \mathrm{mg}, 0.69 \mathrm{mmol})$ and $\mathrm{Pd} / \mathrm{C}(21$ $\mathrm{mg}, 10 \% \mathrm{w} / \mathrm{w} \mathrm{Pd})$ in $\mathrm{MeOH}(20 \mathrm{~mL})$ was degassed (three cycles of freeze, pump and thaw) and then stirred under an atmosphere of $\mathrm{H}_{2}$ ( $1 \mathrm{~atm}$ ) for $16 \mathrm{~h}$ at $23{ }^{\circ} \mathrm{C}$. The mixture was filtered, then the solvent was removed under reduced pressure and the residue was purified by column chromatography (silica gel, $3 \mathrm{~cm} \times 5 \mathrm{~cm}$; EtOAc$\left.\mathrm{MeOH}, 5: 1, R_{f}=0.08\right)$ to yield compound $14(126 \mathrm{mg}, 0.46 \mathrm{mmol}$, $67 \%$ ) as a colorless oil.

$[\alpha]_{\mathrm{D}}^{20}-27.8(c 0.85, \mathrm{MeOH})$.
IR (ATR): 3333 (w), 3055 (w), $2976(w), 2930$ (w), 2867 (w), 1690 (s), $1607(\mathrm{~m}), 1567(\mathrm{~m}), 1494(\mathrm{~m}), 1457(\mathrm{~m}), 1421(\mathrm{~m}), 1366(\mathrm{~s})$, 1273 (s), 1249 (s), 1163 (vs), 994 (w), 979 (w), 744 (w) cm $\mathrm{cm}^{-1}$.

${ }^{1} \mathrm{H}$ NMR (500 MHz, $\left.\mathrm{CD}_{3} \mathrm{OD}\right): \delta=1.33(\mathrm{~s}, 9 \mathrm{H}), 3.16-3.18(\mathrm{~m}, 2 \mathrm{H})$, $3.57(\mathrm{dd}, J=12.4,7.2 \mathrm{~Hz}, 1 \mathrm{H}), 3.84(\mathrm{dd}, J=10.7,7.1 \mathrm{~Hz}, 1 \mathrm{H})$, 4.14 (ddt, $J=10.9,7.0,5.7 \mathrm{~Hz}, 1 \mathrm{H}), 7.42$ (ddd, $J=7.6,4.8,1.1 \mathrm{~Hz}$, $1 \mathrm{H}), 7.53(\mathrm{td}, J=7.7,1.7 \mathrm{~Hz}, 1 \mathrm{H}), 7.93(\mathrm{dt}, J=7.9,1.2 \mathrm{~Hz}, 1 \mathrm{H})$, 8.55 (ddd, $J=4.9,1.6,0.9 \mathrm{~Hz}, 1 \mathrm{H})$.

${ }^{13} \mathrm{C}\left\{{ }^{1} \mathrm{H}\right\}$ NMR (125 MHz, $\left.\mathrm{CD}_{3} \mathrm{OD}\right): \delta=28.7\left(3 \mathrm{CH}_{3}\right), 45.4\left(\mathrm{CH}_{2}\right)$, $53.9\left(\mathrm{CH}_{2}\right), 62.0(\mathrm{CH}), 80.2(\mathrm{C}), 123.7(\mathrm{CH}), 127.1(\mathrm{CH}), 138.3$ $(\mathrm{CH}), 148.4(\mathrm{C}), 150.3(\mathrm{CH}), 158.7(\mathrm{C}), 165.6(\mathrm{C})$.

HRMS (ESI, positive mode): $m / z\left[\mathrm{M}+\mathrm{H}^{+}\right]$calcd for $\mathrm{C}_{14} \mathrm{H}_{21} \mathrm{~N}_{4} \mathrm{O}_{2}$ : 277.1665; found: 277.1659 .

\section{Acknowledgment}

We are grateful to Andre Appeldorn for his assistance in the laboratory.

\section{References}

(1) First reports: (a) Brunner, H.; Obermann, U.; Wimmer, P. J. Organomet. Chem. 1986, 316, C1. (b) Brunner, H.; Obermann, U. Chem. Ber. 1989, 122, 499. (c) Brunner, H.; Obermann, U.; Wimmer, P. Organometallics 1989, 8, 821. (d) Brunner, H.; Brandl, P. J. Organomet. Chem. 1990, 390, C81. (e) Brunner, H.; Wutz, K.; Doyle, M. P. Monatsh. Chem. 1990, 121, 755 .

(2) For reviews, see: (a) Desimoni, G.; Faita, G.; Quadrelli, P. Chem. Rev. 2003, 103, 3119. (b) Aspinall, H. C.; Greeves, N. J. Organomet. Chem. 2002, 647, 151. (c) Johnson, J. S.; Evans, D. A. Acc. Chem. Res. 2000, 33, 325. (d) Nishiyama, H.; Ito, J.-i. Chem. Commun. 2010, 46, 203. (e) Ito, J.-i.; Nishiyama, H. Synlett 2012, 23, 509. (f) Ward, B. D.; Gade, L. Chem. Commun. 2012, 48, 10587.

(3) For recent examples, see: (a) Ingalls, E. L.; Sibbald, P. A.; Kaminsky, W.; Michael, F. E. J. Am. Chem. Soc. 2013, 135, 8854. (b) De Crisci, A. G.; Chung, K.; Oliver, A. G.; SolisIbarra, D.; Waymouth, R. M. Organometallics 2013, 32, 2257. (c) Narayama, A.; Shibatomi, K.; Soga, Y.; Muto, T.; Iwasa, S. Synlett 2013, 24, 375. (d) Holder, J. C.; Marziale, A. N.; Gatti, M.; Mao, B.; Stoltz, B. M. Chem. Eur. J. 2013, 19, 74. (e) Aguado-Ullate, S.; Urbano-Cuadrado, M.; Villalba, I.; Pires, E.; Garcia, J. I.; Bo, C.; Carbo, J. J. Chem. Eur. J. 2012, 18, 14026. (f) Yang, G.; Shen, C.; Zhang, W. Angew. Chem. Int. Ed. 2012, 51, 9141; Angew. Chem. 2012, 124, 9275. (g) Malkov, A. V.; Stewart-Liddon, A. J. P.; McGeoch, G. D.; Ramirez-Lopez, P.; Kocovsky, P. Org. Biomol. Chem. 2012, 10, 4864.

(4) Christoffers, J.; Mann, A.; Pickardt, J. Tetrahedron 1999, 55,5377 .

(5) Maciagiewicz, I. M.; Fang, F.; Roberts, D. A.; Zhou, S.; Hines, J. V.; Bergmeier, S. C. Synthesis 2012, 44, 551.

(6) Griffith, J. A.; Rowlands, G. J. Synthesis 2005, 3446.

(7) Chang, Y.-w.; Yang, J.-j.; Dang, J.-n.; Xue, Y.-x. Synlett 2007, 2283.

(8) Cook, G. R.; Shanker, P. S.; Peterson, S. L. Org. Lett. 1999, 1,615 .

(9) (a) Bhor, S.; Anilkumar, G.; Tse, M. K.; Klawonn, M.; Döbler, C.; Bitterlich, B.; Grotevendt, A.; Beller, M. Org. Lett. 2005, 7, 3393. (b) Tursky, M.; Necas, D.; Drabina, P.; Sedlak, M.; Kotora, M. Organometallics 2006, 25, 901. (c) Ma, K.; You, J. Chem. Eur. J. 2007, 13, 1863. (d) Ohara, M.; Nakamura, S.; Shibata, N. Adv. Synth. Catal. 2011, 353, 3285. (e) Hyodo, K.; Nakamura, S.; Shibata, N. Angew. Chem. Int. Ed. 2012, 51, 10337; Angew. Chem. 2012, 124, 
10483. (f) Barakat, A.; Islam, M. S.; Al Majid, A. M. A.; AlOthman, Z. A. Tetrahedron 2013, 69, 5185. (g) Weber, M.; Klein, J. E. M. N.; Miehlich, B.; Frey, W.; Peters, R. Organometallics 2013, 32, 5810. (h) For a review, see: Liu, H.; Du, D.-M. Adv. Synth. Catal. 2009, 351, 489.

(10) Huang, Y.; Dalton, D. R.; Carroll, P. J. J. Org. Chem. 1997, 62,372 .

(11) Merritt, E. A.; Bagley, M. C. J. Heterocycl. Chem. 2007, 44, 1281.

(12) (a) Shin, C.-g.; Okumura, K.; Ito, A.; Nakamura, Y. Chem. Lett. 1994, 1301. (b) Ayida, B. K.; Simonsen, K. B.;
Vourloumis, D.; Hermann, T. Bioorg. Med. Chem. Lett. 2005, 15, 2457.

(13) Bourland, T. C.; Carter, R. G.; Yokochi, A. F. T. Org. Biomol. Chem. 2004, 2, 1315.

(14) Zaid, F.; El Hajji, S.; El Hallaoui, A.; Elachqar, A.; Kerbal, A.; Roumestant, M. L.; Viallefont, P. Prep. Biochem. Biotechnol. 1998, 28, 137.

(15) Busacca, C. A.; Grossbach, D.; Campbell, S. J.; Dong, Y.; Eriksson, M. C.; Harris, R. E.; Jones, P.-J.; Kim, J.-Y.; Lorenz, J. C.; McKellop, K. B.; O’Brien, E. M.; Qiu, F.; Simpson, R. D.; Smith, L.; So, R. C.; Spinelli, E. M.; Vitous, J.; Zavattaro, C. J. Org. Chem. 2004, 69, 5187. 